\title{
Management of nightmares in patients with posttraumatic stress disorder: current perspectives
}

This article was published in the following Dove Press journal:

Nature and Science of Sleep

\begin{abstract}
Ali A El-Solh ${ }^{1-4}$
'Research Department, VA Western New York Healthcare System, Buffalo, NY, USA; 'Division of Pulmonary, Critical Care, and Sleep Medicine, Department of Medicine, Jacobs School of Medicine and Biomedical Sciences, University of Buffalo, Buffalo, NY, USA; ' ${ }^{\text {Department }}$ of Epidemiology and Environmental Health, School of Public Health and Health Professions, University of Buffalo, Buffalo, NY, USA; ${ }^{4}$ Department of Community and Health Behavior, School of Public Health and Health Professions, University of Buffalo, Buffalo, NY, USA
\end{abstract}

\begin{abstract}
Nightmares are considered the hallmark of posttraumatic stress disorder (PTSD). Although the characteristics of these distressing dreams may vary with the type of traumatic event, the pathophysiology exposes central dysfunction of brain structures at the level of the hippocampus, amygdala, and locus coeruleus, modulated by neurochemical imbalance in noradrenergic, dopaminergic, and serotonin pathways. Underlying comorbid conditions, including other sleep disorders, may contribute to worsening symptoms. Addressing sleep disruption can alleviate the severity of these nocturnal events and augment the effectiveness of other PTSD treatments. The expansion of behavioral treatment modalities for PTSD-related nightmares has been encouraging, but the core of these interventions is heavily structured around memory manipulation and imagery rescripting. A lack of a standardized delivery and a high dropout rate continue to pose significant challenges in achieving successful outcomes. The efficacy of existing pharmacological studies, such as $\alpha$-adrenergic blocking agents, antidepressants, and atypical antipsychotics, has been undermined by methodological limitations and absence of large randomized controlled trials. This review is aimed at reviewing the available treatment strategies for alleviating nightmares in subjects with PTSD. Given the intricate relationship between PTSD and nightmares, future clinical trials have to adopt a more pragmatic approach focused not only on efficacy of novel interventions but also on adjunctive iteration of existing therapies tailored to individual socio-cultural background.
\end{abstract}

Keywords: nightmares, posttraumatic stress disorder, cognitive-behavioral therapy, pharmacotherapy, image rehearsal therapy, prazosin, cannabis

\section{Introduction}

According to the fifth edition of the Diagnostic and Statistical Manual of Mental Disorders, ${ }^{1}$ nightmares, which are considered REM parasomnias, are defined as extreme dysphoric dreams precipitated by a life-threatening event or a perceived harm of individual's emotional and/or physical sense of safety. Not surprisingly, recurrent nightmares are a central feature of posttraumatic stress disorder (PTSD) among both military combat veterans and trauma-exposed civilians. ${ }^{2}$ While the majority of individuals afflicted with PTSD experience sleep dysfunction, the prevalence of posttraumatic nightmares in patients with PTSD can be as high as $72 \% .^{3}$

The exact relation between nightmares and PTSD is still unknown but nightmares and PTSD are closely related, and both show altered activity in the same brain regions. ${ }^{4}$ These nightmares can occur in all stages of sleep but most frequently during the latter part of the night. In most instances, the nightmares are described as replicative in nature where the trauma is being re-enacted. ${ }^{5}$ Awakenings are often accompanied by

Nature and Science of Sleep 2018:10 409-420 
an intense and prolonged sensation of fear or anxiety which causes delayed return to sleep. These patterns of nocturnal sleep disruptions are often associated with excessive somnolence, fatigue, difficulties in concentration, irritability, and feelings of helplessness, which have a detrimental effect on the individual's quality of life. ${ }^{6}$ Irrespective of the triggering events, nightmares often persist for many decades and can be resistant to existing therapy. Among the factors implicated in the importunity of nightmares after trauma are persistence thoughts of helplessness and lack of control, fear and hyperarousal, and REM disruption. ${ }^{7}$

In this mini-review, we examine the characteristic manifestations of nightmares in PTSD. We review studies exploring the health risks attributed to the co-occurrence of nightmares and PTSD. We summarize the existing literature on the psychological and pharmacologic treatments of nightmares in patients with PTSD. Finally, we discuss the challenges encountered in the management of these disorders and identify future research needs.

\section{Methods}

A literature search using Scopus and MEDLINE (1975July 2018) with the terms: nightmares and posttraumatic stress disorder was performed. Inclusion criteria consisted of any clinical trial, retrospective review, or case report of pharmacological or behavioral treatment for nightmares. Studies were required to be written in English to be included. A separate, additional search of PubMed identified articles published electronically prior to print publication and not available through MEDLINE. The clinical trials registries clinicaltrials.gov and Current Controlled Trials (controlled-trials.com) were also queried for unpublished data.

\section{Impact of nightmares on PTSD}

Chronic nightmares in PTSD are associated with adverse consequences and decreased psychological and physiological functioning as well as disturbed sleep. ${ }^{8}$ PTSD and nightmares are intertwined in such a manner that nightmares strengthen PTSD symptoms, and PTSD in turn causes nightmares. However, the presence of nightmares not only influences the development of PTSD but also accelerates the progression of PTSD following trauma exposure. ${ }^{9,10}$ Subjects who reported nightmares prior to trauma exhibited more severe PTSD symptoms after being exposed to a traumatic event than those who did not. Moreover, having nightmares shortly following a traumatic event predicts more severe PTSD symptoms 6 weeks later. ${ }^{11}$ Even with PTSD symptoms abating, nightmares can persist a lifetime.
The distressing nature of these nightmares occasionally results in the display of avoidance behavior before bedtime such as staying late or leaving the lights on at night, which ultimately lead to insomnia and daytime dysfunction. ${ }^{12,13}$ What is more alarming is the propensity of nighttime sufferers to experience frequent suicidal ideas or even to commit an act of suicide. ${ }^{14}$ One study had linked nightmares to a fivefold increase in suicidality. ${ }^{15}$ Furthermore, PTSD substantially heightens the risk of suicidal behaviors, ${ }^{16}$ with one large population-based study indicating that individuals with PTSD were almost three times more likely to experience suicidal ideation than those without PTSD. ${ }^{17}$ Despite the interrelationships between nightmares, PTSD, and suicide, the mechanism by which nightmares confer suicidality has not been identified; however, the common denominator linking these entities together has been ascribed to the sense of entrapment, defeat, and hopelessness. ${ }^{18}$

\section{Approach to management}

Selecting the most appropriate treatment strategy for treating nightmares in context of PTSD should take into account the patient's life experience. A thorough assessment of subclinical psychiatric ailments and sleep disturbances should be sought during initial encounter with health care providers. Recent statistics indicate that nightmares among veterans are largely underreported, as only $11 \%$ to $38 \%$ acknowledged discussing these events with their health care provider. Further, only one-third of adults who verbalized clinically significant nightmares believed that nightmares were a treatable condition. ${ }^{19}$ Given the established risk of behavioral and mental disorders in military recruits returning from combat theaters, screening for nightmares prior to or soon after deployment offers the best opportunity to mitigate the progression of PTSD.

There is currently no objective instrument to measure nightmare content or frequency. Available tools such as dream diaries and questionnaires are self-reported and can be susceptible to subjective bias. Polysomnography (PSG) tracings of posttraumatic nightmares are usually not specific and include increased REM latency, reduced slow-wave sleep, and sleep fragmentation. ${ }^{20}$ These changes are associated with respiratory or leg movement events and increase in heart rate on awakening. ${ }^{21}$ Nonetheless, PSG may be warranted when obstructive sleep apnea is suspected as improvement in sleep quality and nightmares frequency has been reported after continuous positive airway pressure treatment. ${ }^{22}$

Once a determination is made to treat nightmares, the optimal therapeutic intervention (behavioral vs pharmacologic) is a decision shared between the patient and his/her 
clinical provider taking into consideration the severity of nightmares, the duration of treatment, and accessibility to the treatment modality.

\section{Behavioral therapy}

Behavioral treatment of nightmares is based on the concept that nightmares are learned (acquired) behaviors which are amenable to modification with targeted intervention. Traumarelated nightmares are thought to reflect initially an adaptive mechanism attached to the emotional processing of traumatic events but subsequently are converted into a learned habit via classical conditioning. ${ }^{23,24}$

\section{Imagery rehearsal therapy (IRT)}

Introduced by Isaac Marks in 1978, IRT is founded on the premise that the conditioned relationship between nightmares and sleep can be altered via daytime rehearsal of altered dream scripts. ${ }^{25}$ With IRT, the script of the recurring nightmare is modified into a new scenario with a different ending. ${ }^{26}$ The new script is rehearsed daily while awake with the aim of extinguishing the frightening nightmare arousing stimuli while establishing alternative nonfrightening appraisals.

IRT is considered the preferred empiric treatment for posttraumatic nightmares. ${ }^{27,28}$ It can be delivered in group or individual format. The course of treatment is typically completed in less than 12 weeks; however, treatment benefits have been documented after single session group intervention. The efficacy of IRT in cases with PTSD was first demonstrated in a randomized controlled trial of survivors of sexual assault. ${ }^{29}$ Treatment consisted of three sessions (two 3-hour sessions 1 week apart and 1-hour follow-up 3 weeks later) using cognitive imagery treatment presented in a group format. Compared to the control group, IRT resulted in $60 \%$ reduction of disturbing dreams and PTSD symptoms including intrusion, avoidance, and hyperarousal with moderate effect sizes for the treatment (mean Cohen $d=0.60$ ). Therapeutic benefits were sustained between 3 and 6 months without further intervention despite the large dropout rates (32\%). Other studies showed further amelioration in anxiety and somatization with IRT ${ }^{30}$ although the response was less pronounced in PTSD than when treating nightmares in the context of depression. ${ }^{31}$

The application of IRT to veterans with PTSD revealed comparable results with significant decrease in nightmare frequency, nightmare intensity, and depression with treatment effect sizes in the moderate to large range. ${ }^{32,33}$ However not all investigations involving veterans with PTSD have yielded positive results with IRT. Two studies have not been able to reproduce the observed treatment benefits in nightmare frequency, PTSD symptoms, or sleep quality immediately posttreatment albeit a delayed effect emerged 6 months later. ${ }^{34,35}$ Differences in intensity of treatment and presence of comorbidities in the selected population such as coexisting insomnia or sleep-disordered breathing may have influenced study outcomes. Other contributory factors may have been related to the lack of adherence to treatment protocol whether because of missing therapeutic sessions or failure to complete home assignment. Not uncommonly, participants tend to select less stressful events to avoid recollection of distressful nightmares. Despite these caveats, recent systematic reviews and meta-analyses have reiterated the benefits of IRT in decreasing nightmare frequency and distress while improving sleep quality. ${ }^{36,37}$ Adverse effects related to IRT included exacerbation of PTSD symptoms and worsening of negative, distracting, or intrusive images.

The decision to implement IRT for nightmares should be made within the context of comprehensive treatment of PTSD and coexisting sleep disorders. Occasionally, delay in IRT may be warranted in subjects undergoing trauma-focused cognitive-behavioral therapy (CBT) for PTSD. The level of engagement required from participants in these intensive and emotionally charged protocols may interfere with adherence to additional form of behavioral interventions. ${ }^{38}$ Further, there is evidence to suggest that trauma-focused CBT may be associated with resolution of nightmares that even with those patients experiencing residual sleeping dysfunction, less than a quarter reports clinically significant nightmares. ${ }^{39}$

\section{Exposure, rescripting, and relaxation therapy}

Variants of imagery rescripting interventions have been developed since the late 1970s for the treatment of idiopathic nightmares. The exposure, relaxation, and rescripting therapy (ERRT) involves psychoeducation about sleep and nightmares, relaxation, sleep hygiene, exposure, and nightmare rescripting. ${ }^{40}$ While IRT focuses on changing the nightmares by building imagery skills, the exposure element of the ERRT protocol requires individuals to write down their target nightmare and read it aloud so that the event evokes an affect similar to that experienced in the presence of the actual stimulus. The exposure element offers the opportunity for the subject to face the fear in a safe place and habituate to the anxiety.

ERRT is administered through a three-session treatment protocol. ${ }^{41}$ Each session is $\sim 60$ minutes and starts by providing psychoeducation about trauma, nightmares, and sleep 
hygiene, as well as teaching progressive muscle relaxation. The main emphasis is to provide relevant background information, modify maladaptive sleep habits, and help patients gain proficiency with relaxation skills, such as progressive muscle relaxation. Next, treatment focuses on rescripting the nightmare while introducing a new relaxation skill. Patients are to practice these relaxation skills at least twice daily using diaphragmatic breathing for 10 minutes, review the rescripted nightmare using imagery for $\sim 15$ minutes, and engage in progressive muscle relaxation afterward before going to bed. Nightmare rescription combined with daily imagery rehearsal is thought to embed the corrective scenario into the network of fear. In the last session, emphasis is placed on reviewing treatment progress and on discussing maintenance and relapse prevention strategies.

The efficacy of ERRT has been established in multitude of RCTs, case series, and effectiveness studies involving combat veterans, life-threatening accidents, and sexual assault victims with PTSD. ${ }^{41-43}$ Despite the variability in delivery format and treatment structure, a reduction of symptoms of PTSD, depression, and severity of nightmare was consistently observed. Recent meta-analyses confirmed that ERRT yielded larger effect sizes compared with other psychological interventions and pharmacological treatments. ${ }^{27,44}$ The response to ERRT also included amelioration in physiologic reactivity to nightmare imagery. ${ }^{45}$ While the underlying mechanisms for the ERRT-observed responses are not well understood, it is posited that nightmare-related imagery leads to defensive reactivity and autonomic arousal which interferes with sleep quality. ${ }^{42}$ With ERRT intervention, sleep recuperation would reestablish normality to sympathetic output causing a reduction in heart rate reactivity. Not unlike IRT, the extent to which the efficacy of ERRT imparts psychological benefits over analogous behavioral therapy remained undetermined. A recent dismantling study of ERRT whereby participants with trauma-related nightmares were randomized to either ERRT with nightmare exposure and rescripting (NER) or to ERRT without NER found no difference in nightmare frequency, PTSD severity, or sleep quality between the two groups. ${ }^{46}$ While the dose of exposure may not have been sufficient to extinguish nightmare imagery to induce a symptomatic difference, it is plausible that the psychoeducation about trauma, sleep habit modification, and relaxation training are more than adequate in a subgroup of patients who avoid treatment due to nightmare recollection. This abbreviated protocol may offer a promise of an effective relief of these distressing symptoms if validated on a larger scale.

\section{Systematic desensitization}

Systematic desensitization, pioneered by Joseph Wolpe, consists of three essential components. ${ }^{47}$ This form of therapy attempts first to establish a hierarchy of anxiety promoting stimuli. The subject is then expected to assimilate a set of relaxing techniques including synchronization of breathing with relaxation of different muscle groups. The last stage is focused on pairing the relaxation response to the stimuli on the anxiety hierarchy, starting with the lowest level of anxiety. After repeated pairings, the anxiety is expected to lessen in severity, and the relaxation procedure is applied to the next item on the anxiety hierarchy list. If the anxiety is not diminished at a particular step, additional stimuli of lesser anxiety level are developed to bridge the gap. This procedure is repeated through all items on the hierarchy ladder leading ultimately to elimination of these distressing memories. ${ }^{48}$

Three studies have evaluated systematic desensitization in the treatment of nightmares. ${ }^{49,50}$ In comparison with muscle relaxation or continuous self-recording, systematic desensitization resulted in decreased nightmares frequency and/or intensity up to 7-month follow-up. However, these studies were silent on whether the diagnosis of PTSD was established in the study samples, thus limiting the generalizability of these findings to the PTSD population.

\section{Eye movement desensitization and reprocessing (EMDR)}

EMDR therapeutic approach is guided by the Adaptive Information Processing Model whereby nightmares are viewed as manifestations of disturbing events that have not been adequately processed but encoded and stored in a state-specific dysfunctional form. ${ }^{51}$ EMDR therapy elicits the recall of these distressing images while activating one type of bilateral sensory input such as hand tapping or side to side eye movement. By overloading the working memory, emotional reactivity is dampened and imagery becomes less vivid. It is speculated that this technique produces emotional and physiologic alterations resulting in a relaxing response including respiratory synchronization, reduction in heart rate, and reduction in galvanic response. ${ }^{52}$

EMDR has been increasingly administered to patients with PTSD with promising results. ${ }^{53}$ Although it has been suggested that EMDR may be more effective than CBT in decreasing the severity of intrusion and arousal symptoms, the efficacy of this intervention on PTSD-associated nightmares has not been thoroughly validated in randomized controlled trials. However, a case series of 83 Vietnam veterans with PTSD compared EMDR with Relaxation Training and Biofeedback on initial admission to an inpatient PTSD 
program, with a follow-up assessment at 60 and 90 days later. Veterans who received EMDR described a reduction in nightmare severity to a higher extent that the competing intervention. ${ }^{54}$ No adverse effects were reported but it was unclear how many of those participants completed treatment or dropped out from the study.

\section{Lucid dreaming therapy}

The term lucid dreaming treatment (LDT) was coined by Van Eeden in $1913 .{ }^{55}$ It is considered an alternative to cognitive restructuring techniques. Unlike IRT, LDT appears to target nightmares exclusively without affecting PTSD symptoms. The intervention consists of nightmares sufferers becoming lucid to their nightmares through various daily exercises such as questioning the nature of the environment "Is this real or am I dreaming?" Once lucidity is established, it is anticipated that the fear from the nightmares becomes less intense because the dreamer can re-experience the traumatic event while having more control and less fear. The success of LDT hinges on the assumption that dream recall will ensue and that the subjects will become lucid during the intervention phase. In a cross-sectional study of 32 veterans with PTSD, 88\% of participants reported at least one nightmare per week. ${ }^{56}$ While $82 \%$ had frequent awareness of nightmares, only $24 \%$ had nightmare content control indicating potential role of LD therapy in controlling distressing dreams. However, the efficacy of LD over other cognitive-behavioral therapy for PTSD-related nightmares has not been established.

\section{Cognitive-behavioral treatment for insomnia}

Given that nightmares are frequently described in the setting of insomnia, treatment protocols have incorporated CBT for insomnia (CBT-I) with CBT for nightmares. A meta-analysis of RCTs involving various cognitive-behavioral modalities for both conditions concluded that mixed CBT-I and IRT interventions were associated with larger effects on sleep quality than IRT interventions alone. ${ }^{36}$ However, the inclusion of CBT-I did not further ameliorate nightmares or PTSD symptoms. Further, protocols that consisted of nightmare exposure and/or relaxation training, such as ERRT or imagery rescripting and exposure therapy, were as effective as IRT protocols that did not include these elements, suggesting that not all components may be necessary for clinical recovery. ${ }^{36}$ These studies highlight the need for future research exploring various combinations of techniques to determine the optimal approach for addressing trauma nightmares.

\section{Pharmacological therapy}

The limited evidence base for the pharmacotherapy of nightmares constitutes a major challenge to the effective treatment of this parasomnia. The currently prescribed medications for the management of nightmares lack the rigorous validity of multisite Phase III trials to support a strong recommendation for any of the available agents.

\section{Sympatholytic medication}

The use of $\alpha-1$ adrenergic receptor antagonists in the treatment of PTSD-associated nightmares is predicated on the fact that consolidation of emotional memories is facilitated by the activation of $\alpha-1$ adrenergic receptors. After each retrieval, an established memory requires stabilization (or reconsolidation) to be re-established for its later access. ${ }^{57}$ Following retrieval of conditioned fear, noradrenergic stimulation mediates memory reconsolidation and confers memory resistance against fear extinction. ${ }^{58}$ Alpha-adrenergic blockade attenuates the negative valence associated with traumatic memories and disrupts the process of fear recollection. ${ }^{59}$

Prazosin, a lipid soluble $\alpha-1$ adrenergic receptor antagonist, was first introduced in 1970s as a U.S. Food and Drug Administration (FDA)-approved agent for the treatment of hypertension. Although rarely used today for this indication, prazosin has emerged as the leading agent for the treatment of PTSD-related nightmares because of its putative role in disrupting consolidation of fear memories and reducing primitive fear response. ${ }^{59}$ Numerous case reports, open-label trials, and RCTs have shown the efficacy of prazosin in treating nightmares of patients with PTSD ${ }^{60-64}$ A meta-analysis of six RCTs comparing prazosin to placebo found a significant reduction in nightmares distress and/or frequency as well as overall PTSD symptoms. ${ }^{65}$ The average daily dose ranged between 8 and $20 \mathrm{mg}$ /day. Civilians with PTSD-related nightmares required lower doses with an average of $3.1 \pm 3 \mathrm{mg} /$ day. In these studies, the duration of treatment lasted between 7 and 20 weeks but the time to clinical improvement varied between patients due to heterogeneity in studies' inclusion criteria, coexistent psychiatric comorbidities, and concurrent medications. A more recent RCT, prazosin for combat trauma PTSD (PACT), failed to establish the efficacy of prazosin in reducing nightmare frequency and intensity in military veterans with PTSD. ${ }^{66}$ Both at 10 and 26 weeks, there was no significant difference between the prazosin and the placebo group in self-reported recurrent distressing dreams and overall sleep quality. These disappointing results were attributed to selection bias related to the inclusion of clinically stable patients who were unlikely to respond to adrenergic blockade and possibly to sample 
contamination by participants who had undiagnosed sleep apnea. Inadequate drug exposure was also considered among the reasons for the negative findings. While the dose that the participants received ranged between 2.0 and $20.0 \mathrm{mg} /$ day, a handful of case reports have reported successful resolution of nightmares with higher doses of prazosin $(25-45 \mathrm{mg}){ }^{63,67}$ It is noteworthy that incidence of new or worsening suicidal ideation in the PACT trial was less common in the prazosin group than in the placebo group. Given the unpredictable response rate, a recent position paper from the American Academy of Sleep Medicine (AASM) downgraded the level of recommendation of prazosin while maintaining its status as the first choice pharmacologic agent for the treatment of nightmares ${ }^{68}$ The most significant adverse reaction to prazosin is the first dose phenomenon characterized by precipitous drop in blood pressure and sudden loss of consciousness when given at $2 \mathrm{mg}$ or greater. This can be minimized by limiting the initial dose to $1 \mathrm{mg}$ followed by gradual increase until the desired effect is achieved or the maximum tolerable dose is attained. Other reported side effects of prazosin include dizziness $(10 \%)$, headache (8\%), drowsiness ( $8 \%$ ), and lack of energy ( $8 \%)$.

Terazosin is another $\alpha-1$ adrenergic antagonist that has been reported to reduce nightmares associated with PTSD. ${ }^{69,70}$ While terazosin did not achieve full recall cessation, reduction of symptoms was described on $64 \%$ of the trials with dose ranging between 2.0 and $5.0 \mathrm{mg} .{ }^{71}$ Similarly, doxazosin (1.0-6.0 mg) has shown promise in PTSD nightmares. ${ }^{72,73}$ Because of improved absorption profile and longer half-life and its availability as an extended release, doxazosin may offer significant advantages over prazosin in clinical practice. However, none of these agents had been rigorously examined in a RCT.

Clonidine, an $\alpha-2$ adrenergic receptor agonist has been reported to decrease nightmares in patients with comorbid PTSD and traumatic brain injury. ${ }^{74}$ It suppresses sympathetic nervous system outflow at the locus coeruleus by binding to presynaptic adrenergic receptors. Data supporting the use of clonidine are limited to case series with the most compelling evidence originating from its use in Cambodian refugees. ${ }^{75,76}$ When augmented with imipramine $(150 \mathrm{mg} /$ day $)$, clonidine $(0.2 \mathrm{mg} /$ day $)$ resulted in reduction of nightmares in seven of the nine participants. A more recent chart review of 76 veterans diagnosed with PTSD who received either clonidine or prazosin documented $57 \%$ and $53 \%$ positive response for nighttime PTSD symptoms within 6 months of initiating therapy, respectively. ${ }^{77}$ Two years after starting clonidine, only eight remained on therapy raising questions about sustained benefit. The design of the study did not examine, however, the potential interaction between antidepressants and $\alpha-2$ adrenergic agents which could have impacted clinical outcomes.

\section{Antipsychotics}

Several antipsychotics have been studied off-label for nightmares with promising effects on treatment outcomes. However, most of the evidence from studies evaluated in the AASM's Best Practice Guide are considered to be low quality. ${ }^{68}$ Aside from the lack of high quality studies, antipsychotics are associated with serious adverse side effects including excessive sedation, restless leg syndrome, cognitive dysfunction, and metabolic derangements. ${ }^{78}$ Table 1 shows the affinity of several antipsychotics for the neurotransmitters and represents global receptor affinity based on different studies, showing the large variation in pharmacological properties of frequently used antipsychotics in the management of PTSDrelated nightmares.

Risperidone is an atypical antipsychotic with high affinity for serotonin receptors and a lower affinity for dopamine-2 receptor. ${ }^{79}$ It also exhibits significant $\alpha-1$ and $\alpha-2$ noradrenergic antagonism. ${ }^{80}$ These unique pharmacologic properties permit risperidone to modify not only serotoninergic, dopaminergic, and noradrenergic neurotransmission, but also to

Table I Receptor-binding profiles of antipsychotics prescribed in the treatment of PTSD nightmares

\begin{tabular}{|c|c|c|c|c|c|c|c|c|}
\hline & $\mathbf{D}_{1}$ & $\mathbf{D}_{2}$ & $5 \mathrm{HT}_{\mathrm{IA}}$ & $5 \mathrm{HT}_{2 \mathrm{~A}}$ & $\mathbf{5 H T}_{2 \mathrm{C}}$ & $\alpha_{1}$ & $\alpha_{2}$ & $\mathbf{H}_{1}$ \\
\hline \multicolumn{9}{|c|}{ Typical antipsychotics } \\
\hline Thioridazine & ++ & ++ & + & ++ & ++ & +++ & 0 & ++ \\
\hline Perphenazine & + & ++++ & + & +++ & + & ++ & + & +++ \\
\hline \multicolumn{9}{|c|}{ Atypical antipsychotics } \\
\hline Olanzapine & ++ & ++ & 0 & +++ & ++ & ++ & + & ++++ \\
\hline Quetiapine & + & + & + & + & 0 & ++ & + & ++ \\
\hline Ripseridone & ++ & +++ & + & ++++ & ++ & +++ & +++ & +++ \\
\hline Aripiprazole & + & ++++ & +++ & ++ & ++ & ++ & ++ & ++ \\
\hline
\end{tabular}

Notes: Affinity for receptors: $0=$ absent; +=low; ++=moderate; $+++=$ high; $++++=$ very high. $5 \mathrm{HT}_{1 \mathrm{~A}}, 5 \mathrm{HT}_{2 \mathrm{~A}}$, and $5 \mathrm{HT}_{2 \mathrm{C}}$ are known as serotonin receptors. Abbreviations: $\alpha$, alpha-adrenergic receptor; $\mathrm{D}$, dopamine receptor; $\mathrm{H}$, histamine receptor; $5 \mathrm{HT}$, 5 -hydroxytryptamine; PTSD, posttraumatic stress disorder. 
modulate the prefrontal cortex and the amygdala which are dysregulated in PTSD-related sleep disturbances, including nightmares.

Three studies have evaluated the response of risperidone in patients with PTSD-associated nightmares. ${ }^{81-83}$ A preliminary retrospective study of ten patients hospitalized for severe burns reported symptomatic improvement in flashbacks/ nightmares after receiving an average of $1 \mathrm{mg}$ of risperidone. ${ }^{82}$ These findings were replicated in a pilot, open-label trial, involving twenty Vietnam combat male veterans who were on stable doses of antipsychotropic medications. The regimen included a flexible dose of adjunctive risperidone (mean maximum dose $2.3 \pm 0.6 \mathrm{mg}$ ) for 12 weeks. ${ }^{81}$ Of the 17 participants who completed the study, marked improvement was noted in the recurrent distressing dreams as early as 6 weeks in the trial. There was also a significant reduction in the proportion of diaries reporting nightmares. The question of whether risperidone is effective as a dedicated treatment for PTSD-related nightmares was addressed in a more recent retrospective study of 65 combat veterans. ${ }^{83}$ Risperidone dosing ranged from 0.5 to $4.0 \mathrm{mg} /$ day. Full nightmare cessation was reported in $43 \%$ of patients with another $41.5 \%$ reporting a decrease in the frequency and intensity of nightmares. No correlation was observed between the risperidone dose and the treatment effect.

Olanzapine and quetiapine have been used either as a single agent or as an adjunct treatment for sleep disorders, PTSD symptoms, and insomnia in combat veterans. These atypical antipsychotics possess antidopaminergic and antiserotonergic properties and exhibit high affinity for $\alpha-1$ and histamine $\mathrm{H} 1$ receptors. A series of case reports indicated that the addition of olanzapine to selective serotonin reuptake inhibitor (SSRI) showed promise in ameliorating nightmares and insomnia complaints with patients with PTSD. ${ }^{84}$ These findings corroborated the results of a double-blind placebocontrolled study in which olanzapine augmentation with SSRI was associated with greater reduction in specific measures of PTSD and nightmares than placebo. ${ }^{85}$ However, another double-blind study reported no difference between olanzapine monotherapy and placebo. ${ }^{86}$ In these studies, olanzapine was associated with weight gain to a greater extent than the comparator group due to occupancy of the histamine receptors and changes to neurochemical signaling in regions of the brain that regulate appetite. ${ }^{87,88}$ Similarly, quetiapine (25-300 mg) showed significant albeit modest reduction in nightmares distress in combat veterans with PTSD. ${ }^{89}$ However, even when the dosing was escalated to 800 $\mathrm{mg} /$ day, there were as many failed trials as successful trials. ${ }^{71}$
Aripiprazole with its high affinity for the Dopamine-2 and D3 receptors and its partial agonist/antagonist activity at the serotonin $5-\mathrm{HT}_{1 \mathrm{~A}}$ binding sites has been proven an effective agent for the treatment of psychotic and mood disorders. ${ }^{90}$ Five cases of combat-related PTSD treated with aripiprazole (dose $15-30 \mathrm{mg} /$ day) in combination with either sertraline or cognitive-behavioral psychotherapy showed a significant improvement, but not total resolution, of nightmares..$^{91}$ Only one patient discontinued treatment due to worsening insomnia. Aripiprazole could be a reasonable option owing to its lower rate of weight gain and elevated prolactin compared with the other atypical antipsychotic medications. It should be noted, however, that none of these agents have received FDA approval for the treatment of nightmares.

Of the typical antipsychotics, thioridazine and perphenazine have been the subject of case reports addressing the treatment of PTSD-related nightmares. ${ }^{71,92}$ These reports are limited by small sample size and methodological flaws. Not unlike the atypical antipsychotic agents, the evidence for their use is inconclusive and not recommended for this condition.

\section{Antidepressants}

SSRIs are considered first-line treatment for all four-symptom clusters associated with PTSD. While there is evidence that SSRIs may attenuate the severity of nightmares, the response to these agents has not been consistent. Expert opinion has cited paroxetine and citalopram to be partially successful in alleviating nightmares distress at doses of 60 and $40 \mathrm{mg}$, respectively, but none of these agents have been examined in a randomized trial. ${ }^{71}$ Two small open-label studies have alluded to the benefits of fluvoxamine in managing PTSD nightmares. A 12-week prospective study in a group of 24 Dutch veterans with PTSD reported modest improvement in self-reported nightmares after receiving fluvoxamine (50-300 mg/day). ${ }^{93}$ However, $50 \%$ of the participants did not complete the study, of whom nine dropped out because of gastrointestinal symptoms. These findings were reiterated in another open-label trial involving 21 Vietnam combat veterans with PTSD. ${ }^{94}$ Fluvoxamine intake (50-250 mg/day) was translated into improvement of all domains of subjective sleep quality. The largest effect seen was for "dreams linked to traumatic experience" but "bad dreams" as measured by the Stress Reporting Rating Scale showed only marginal benefit. No adverse reaction was reported in that study. It is noteworthy to mention that multiple case reports have implicated SSRIs such as venlafaxine, fluoxetine, ${ }^{95}$ and citalopram $^{96}$ in exacerbating nightmares. At this time, existing 
evidence does not support the use of SSRIs as a dedicated treatment for PTSD-associated nightmares.

Antidepressants with antihistaminergic properties, such as mirtazapine, or antagonistic action at serotonergic $5-\mathrm{HT}_{2}$ receptors, like nefazodone and trazodone, have been used in the treatment of nightmares in patients PTSD with varying success. Mirtazapine, a selective $\alpha$ - 2 adrenergic blocker and specific serotonergic antidepressant, was initially reported to decrease frequency and intensity of nightmares in more than 200 refugees. ${ }^{97}$ However, the experience reported from a VA medical center showed a failure rate of $50 \%$ with the other $50 \%$ describing partial or complete response. ${ }^{71}$ The dose ranged from 7.5 to $45 \mathrm{mg}$ daily. Paradoxically, mirtazapine has been linked to new onset of nightmares. ${ }^{98,99}$ This adverse event is attributed to the fact that mirtazapine, unlike other antidepressants, promotes REM sleep together with shortening of sleep onset, increased total sleep time, and slow-wave sleep. In addition, mirtazapine may induce restless legs syndrome in as many as $28 \%$ of patients. ${ }^{100}$

Studies using nefazodone for PTSD nightmares have yielded positive outcomes at doses of 50-600 mg ${ }^{101-103}$ but concerns about life-threatening hepatotoxicity have made this agent less desirable and the last position paper by the AASM for the treatment of nightmare disorder in adults has dropped this agent from its listing. ${ }^{68}$ Trazodone remains the most prescribed PTSD anti-nightmare agent in the setting of insomnia because of its low risk of abuse and dependence. Trazodone is a potent antagonist of serotonin $5 \mathrm{HT}_{2 \mathrm{~A}}$ and $5 \mathrm{HT}_{2 \mathrm{C}} /$ histaminergic receptors with a moderate-to-highly potent $\alpha$-adrenergic receptor antagonist. Due to its sedative properties, trazodone has been used in the treatment of insomnia and nightmares. A report from Palo Alto Veterans Affairs Health Care System that included veterans seeking in patient treatment for PTSD, trazodone decreased nightmares frequency in $72 \%$ from an average of 3.3 nights per week to 1.3 nights per week. ${ }^{104}$ There was a significant correlation between the effectiveness in decreasing nightmares and improving sleep. The effective dose range of trazodone for $70 \%$ of patients was $50-200 \mathrm{mg}$ nightly. Side effects included daytime sedation, dry mouth, dizziness, and priapism, which led to discontinuation of the drug in $19 \%$ of participants.

\section{Other agents}

Cyproheptadine has been described in anecdotal series as an effective agent for the treatment of nightmares. ${ }^{105,106}$ Its efficacy in reducing nightmares has been attributed to REM suppression due to its antagonistic effect at the serotonin autoreceptor $\left(5-\mathrm{HT}_{1 \mathrm{~A}}\right)$, resulting in increased serotonin outflow at the midbrain raphe. Cyproheptadine possesses also strong anticholinergic properties, which have been linked to reduction of REM sleep. ${ }^{107} \mathrm{~A}$ small case series of four veterans out representing a group of 80 with combat-related PTSD selfreported elimination of nightmares in $75 \%$ of participants within few days after receiving 16-24 mg of cyproheptadine. ${ }^{106}$ Another retrospective study detailed the response of nine patients with PTSD who had partial to full resolution of nightmares within 3-4 weeks of cyproheptadine initiation at doses ranging from 4 to $12 \mathrm{mg} /$ day. Although patients subjectively recounted a reduction in nightmares, the total number of records reviewed was not reported. More recently, a large open-label augmentation trial with cyproheptadine of 32 veterans with recurrent distressing dreams showed no significant improvement in any of the outcomes related to the presence of dreams, content of dreams, or disturbance of dreams. ${ }^{108}$ Cyproheptadine was not well tolerated, as $28 \%$ of the patients discontinued the drug as a result of side effects.

Benzodiazepines and non-benzodiazepines receptors are commonly prescribed for PTSD-related hyperarousal and anxiety disorders ${ }^{109,110}$ despite the fact that benzodiazepine receptor agonists are considered relatively contraindicated for patients with PTSD or recent trauma. ${ }^{111}$ In line with these recommendations, RCTs examining the efficacy of clonazepam, alprazolam, and zolpidem with or without psychotropic agents in reducing nightmares have found little evidence to support their use. ${ }^{112-114}$

Recently, anecdotal reports of relief from PTSD sleep disturbances with the use of cannabis ${ }^{115,116}$ have created interest in investigating the role of the endocannabinoid system in PTSD and nightmares. Given the interactions between cannabinoids with neuromodulators, such as dopamine, glutamate, serotonin, gamma-amino-butyric acid, it has been thought that cannabis may potentially confer some degree of medical benefit to posttraumatic stress victims. Cannabinoids have a broad spectrum of action that includes hypnotic, antipsychotic, dream recall suppression, and anxiolytics properties. ${ }^{117}$ Nabilone, a synthetic endocannabinoid receptor (CB1 and $\mathrm{CB} 2$ ) agonist, has been in use in Europe and Canada for over 25 years and has gained FDA approval for the treatment of chemotherapy-induced nausea and vomiting. This synthetic cannabinoid (SC) was initially reported to have resolved or lessened the severity of nightmares in $72 \%$ of cases based on a chart review of 47 patients with at least a 2-year history of PTSD-related nightmares. ${ }^{118}$ Patients was started at $0.5 \mathrm{mg} 1$ hour prior to bedtime and titrated up to $4.0 \mathrm{mg}$. Discontinuation was attempted every 6 months. While four patients had complete response following 4-12 months, the rest experienced 
recurrence of nightmares following nabilone withdrawal necessitating resumption of treatment. Twenty-eight percent discontinued the medication because of side effects. A more recent, double-blind, placebo-controlled trial in 29 Canadian service members with documented PTSD had given additional support for the potential use of SCs for the treatment of PTSD-related nightmares. ${ }^{119}$ The regimen started with $0.5 \mathrm{mg}$ and titrated to a maximum of $3 \mathrm{mg}$. The Clinician-administered PTSD Scale recurring and distressing dream scores were significantly reduced in the nabilone group compared to placebo. These findings were associated with improved overall general well-being. Nabilone was well tolerated. The most frequent side effects were dry mouth and headache. Despite these promising results, long-term use of SCs has not been safely established. Case reports and systematic analyses of SCs have described severe toxic effects of SC including respiratory depression, ${ }^{120}$ hyperthermia, ${ }^{121}$ acute cerebral ischemia, ${ }^{122}$ and seizures. ${ }^{123,124}$ Abrupt discontinuation of daily SC can precipitate withdrawal syndrome three or four days after stoppage characterized by waxing and waning behavioral, mood and physical symptoms such weakness, sweating, restlessness, dysphoria, sleeping problems, anxiety, craving, and diaphoresis. ${ }^{125}$ There have been also reports of association between cannabis use and psychosis with evidence suggesting that cannabis may precipitate psychotic crisis in subjects vulnerable to such illness. ${ }^{126}$ Given the extent of medicinal and recreational use of cannabis, goodquality safety and efficacy data are urgently needed.

\section{Future directions}

The literature to date on the management of nightmares in PTSD is limited by a paucity of randomized controlled study designs and the use of different measures to assess health care outcomes. While significant advances have been made on nightmare-focused CBT such as exposure or systematic desensitization and techniques of cognitive restructuring, much sought long-term comparative effectiveness trials are paramount in formulating practical choices. Other studies should identify predictors of treatment efficacy and examine patient preferences in order to tailor treatment to a particular phenotype or a specific group. A focus on developing standardized intervention protocols along with measures of treatment integrity and fidelity could also provide more information on the efficacy of individual treatment components.

With the disappointing results of the VA Cooperative Study Program trial, combining pharmacotherapy with psychotherapy may produce a large effect size than either treatment modality alone. Because the notion of treating nightmares should be aligned with ongoing management of PTSD, sleep disruptions and suicide, experimentation with multifaceted behavioral and pharmaceutical therapy targeting insomnia and depression should be explored. Lastly, advanced research on complementary and alternative modalities including yoga, acupuncture, and meditation with or without CBT may offer parallel venues for the treatment of nightmares.

\section{Disclosure}

The author reports no conflicts of interest in this work.

\section{References}

1. American Psychiatric Association. Diagnostic and Statistical Manual of Mental Disorders. 5th ed. Washington, DC: American Psychiatric Association; 2003.

2. Germain A. Sleep disturbances as the hallmark of PTSD: where are we now? Am J Psychiatry. 2013;170(4):372-382.

3. Leskin GA, Woodward SH, Young HE, Sheikh JI. Effects of comorbid diagnoses on sleep disturbance in PTSD. J Psychiatr Res. 2002;36(6):449-452.

4. Heim C, Nemeroff CB. Neurobiology of posttraumatic stress disorder. CNS Spectr. 2009;14(1 Suppl 1):13-24.

5. Davis JL, Byrd P, Rhudy JL, Wright DC. Characteristics of chronic nightmares in a trauma-exposed treatment-seeking sample. Dreaming. 2007;17(4):187-198.

6. Detweiler MB, Arif S, Candelario J, et al. Salem VAMC-U.S. Army fort Bragg warrior transition clinic telepsychiatry collaboration: 12-month operation clinical perspective. Telemed J E Health. 2012;18(2):81-86.

7. Davies J. Treating Post-trauma Nightmares: A Cognitive Behavioral Approach. New York: Springer; 2009.

8. Clum GA, Nishith P, Resick PA. Trauma-related sleep disturbance and self-reported physical health symptoms in treatment-seeking female rape victims. J Nerv Ment Dis. 2001;189(9):618-622.

9. Bryant RA, Creamer M, O'Donnell M, Silove D, McFarlane AC. Sleep disturbance immediately prior to trauma predicts subsequent psychiatric disorder. Sleep. 2010;33(1):69-74.

10. van Liempt S, van Zuiden M, Westenberg H, Super A, Vermetten E. Impact of impaired sleep on the development of PTSD symptoms in combat veterans: a prospective longitudinal cohort study. Depress Anxiety. 2013;30(5):469-474.

11. Kobayashi I, Sledjeski EM, Spoonster E, Fallon WF, Delahanty DL. Effects of early nightmares on the development of sleep disturbances in motor vehicle accident victims. JTrauma Stress. 2008;21(6):548-555.

12. Spoormaker VI, Montgomery P. Disturbed sleep in post-traumatic stress disorder: secondary symptom or core feature? Sleep Med Rev. 2008;12(3):169-184.

13. Nadorff MR, Nazem S, Fiske A. Insomnia symptoms, nightmares, and suicide risk: duration of sleep disturbance matters. Suicide Life Threat Behav. 2013;43(2):139-149.

14. Sjöström N, Hetta J, Waern M. Persistent nightmares are associated with repeat suicide attempt: a prospective study. Psychiatry Res. 2009;170(2-3):208-211.

15. Sjöström N, Waern M, Hetta J. Nightmares and sleep disturbances in relation to suicidality in suicide attempters. Sleep. 2007;30(1):91-95.

16. Panagioti M, Gooding PA, Tarrier N. A meta-analysis of the association between posttraumatic stress disorder and suicidality: the role of comorbid depression. Compr Psychiatry. 2012;53(7):915-930.

17. Sareen J, Houlahan T, Cox BJ, Asmundson GJ. Anxiety disorders associated with suicidal ideation and suicide attempts in the National Comorbidity Survey. J Nerv Ment Dis. 2005;193(7):450-454.

18. Littlewood DL, Gooding PA, Panagioti M, Kyle SD. Nightmares and suicide in posttraumatic stress disorder: the mediating role of defeat, entrapment, and hopelessness. J Clin Sleep Med. 2016;12(3):393-399. 
19. Nadorff MR, NadorffDK, Germain A. Nightmares: under-reported, undetected, and therefore untreated. J Clin Sleep Med. 2015;11(7):747-750.

20. Simor P, Horváth K, Gombos F, Takács KP, Bódizs R. Disturbed dreaming and sleep quality: altered sleep architecture in subjects with frequent nightmares. Eur Arch Psychiatry Clin Neurosci. 2012;262(8):687-696.

21. Phelps AJ, Kanaan RAA, Worsnop C, Redston S, Ralph N, Forbes D. An ambulatory polysomnography study of the post-traumatic nightmares of post-traumatic stress disorder. Sleep. 2018;41(1).

22. Tamanna S, Parker JD, Lyons J, Ullah MI. The effect of continuous positive air pressure (CPAP) on nightmares in patients with posttraumatic stress disorder (PTSD) and obstructive sleep apnea (OSA. J Clin Sleep Med. 2014;10(6):631-636.

23. Committee on the Assessment of Ongoing Effects in the Treatment of Posttraumatic Stress Disorder; Institute of Medicine. Treatment for Posttraumatic Stress Disorder in Military and Veteran Populations. Washington, DC: National Academies Press; 2012.

24. Foa EB, Steketee G, Rothbaum BO. Behavioral/cognitive conceptualizations of post-traumatic stress disorder. Behav Ther. 1989;20:155-176.

25. Marks I. Rehearsal relief of a nightmare. Br J Psychiatry. 1978;133(5): 461-465.

26. Krakow B, Zadra A. Clinical management of chronic nightmares: imagery rehearsal therapy. Behav Sleep Med. 2006;4(1):45-70.

27. Augedal AW, Hansen KS, Kronhaug CR, Harvey AG, Pallesen S. Randomized controlled trials of psychological and pharmacological treatments for nightmares: a meta-analysis. Sleep Med Rev. 2013;17(2):143-152.

28. Hansen K, Höfling V, Kröner-Borowik T, Stangier U, Steil R. Efficacy of psychological interventions aiming to reduce chronic nightmares: a meta-analysis. Clin Psychol Rev. 2013;33(1):146-155.

29. Krakow B, Hollifield M, Johnston L, et al. Imagery rehearsal therapy for chronic nightmares in sexual assault survivors with posttraumatic stress disorder: a randomized controlled trial. JAMA. 2001;286(5):537-545.

30. Krakow B, Kellner R, Neidhardt J, Pathak D, Lambert L. Imagery rehearsal treatment of chronic nightmares: with a thirty month followup. J Behav Ther Exp Psychiatry. 1993;24(4):325-330.

31. Thünker J, Pietrowsky R. Effectiveness of a manualized imagery rehearsal therapy for patients suffering from nightmare disorders with and without a comorbidity of depression or PTSD. Behav Res Ther. 2012;50(9):558-564.

32. Forbes D, Phelps AJ, McHugh AF, Debenham P, Hopwood M, Creamer $M$. Imagery rehearsal in the treatment of posttraumatic nightmares in Australian veterans with chronic combat-related PTSD: 12-month follow-up data. J Trauma Stress. 2003;16(5):509-513.

33. Nappi CM, Drummond SP, Thorp SR, McQuaid JR. Effectiveness of imagery rehearsal therapy for the treatment of combat-related nightmares in veterans. Behav Ther. 2010;41(2):237-244.

34. Cook JM, Harb GC, Gehrman PR, et al. Imagery rehearsal for posttraumatic nightmares: a randomized controlled trial. J Trauma Stress. 2010;23(5):553-563.

35. Lu M, Wagner A, Van Male L, Whitehead A, Boehnlein J. Imagery rehearsal therapy for posttraumatic nightmares in U.S. veterans. $J$ Trauma Stress. 2009;22(3):236-239.

36. Casement MD, Swanson LM. A meta-analysis of imagery rehearsal for post-trauma nightmares: effects on nightmare frequency, sleep quality, and posttraumatic stress. Clin Psychol Rev. 2012;32(6):566-574.

37. Harb GC, Phelps AJ, Forbes D, Ross RJ, Gehrman PR, Cook JM. A critical review of the evidence base of imagery rehearsal for posttraumatic nightmares: pointing the way for future research. J Trauma Stress. 2013;26(5):570-579.

38. Manber R, Carney C, Edinger J, et al. Dissemination of CBTI to the non-sleep specialist: protocol development and training issues. J Clin Sleep Med. 2012;8(2):209-218.

39. Zayfert C, Deviva JC. Residual insomnia following cognitive behavioral therapy for PTSD. J Trauma Stress. 2004;17(1):69-73.
40. Davis JL, Wright DC. Exposure, relaxation, and rescripting treatment for trauma-related nighmares. J Trauma Dissociation. 2006;7(1): $5-18$.

41. Davis JL, Wright DC. Randomized clinical trial for treatment of chronic nightmares in trauma-exposed adults. J Trauma Stress. 2007;20(2):123-133.

42. Davis JL, Rhudy JL, Pruiksma KE, et al. Physiological predictors of response to exposure, relaxation, and rescripting therapy for chronic nightmares in a randomized clinical trial. J Clin Sleep Med. 2011;7(6):622-631.

43. Balliett NE, Davis JL, Miller KE. Efficacy of a brief treatment for nightmares and sleep disturbances for veterans. Psychol Trauma. 2015;7(6):507-515.

44. Ho FY, Chan CS, Tang KN. Cognitive-behavioral therapy for sleep disturbances in treating posttraumatic stress disorder symptoms: a meta-analysis of randomized controlled trials. Clin Psychol Rev. 2016;43:90-102.

45. Rhudy JL, Davis JL, Williams AE, et al. Cognitive-behavioral treatment for chronic nightmares in trauma-exposed persons: assessing physiological reactions to nightmare-related fear. J Clin Psychol. 2010;66(4):365-382.

46. Pruiksma KE, Cranston CC, Rhudy JL, Micol RL, Davis JL. Randomized controlled trial to dismantle exposure, relaxation, and rescripting therapy (ERRT) for trauma-related nightmares. Psychol Trauma. 2018;10(1):67-75.

47. Wolpe J. Behavioral Therapy Techniques. New York: Pergamon Press; 1967.

48. Cellucci AJ, Lawrence PS. The efficacy of systematic desensitization in reducing nightmares. J Behav Ther Exp Psychiatry. 1978;9(2):109-114.

49. Kellner R, Neidhardt J, Krakow B, Pathak D. Changes in chronic nightmares after one session of desensitization or rehearsal instructions. Am J Psychiatry. 1992;149(5):659-663.

50. Miller WR, Dipilato M. Treatment of nightmares via relaxation and desensitization: a controlled evaluation. J Consult Clin Psychol. 1983;51(6):870-877.

51. Shapiro F. Eye movement desensitization: a new treatment for post-traumatic stress disorder. J Behav Ther Exp Psychiatry. 1989;20(3):211-217.

52. Wilson DL, Silver SM, Covi WG, Foster S. Eye movement desensitization and reprocessing: effectiveness and autonomic correlates. $J$ Behav Ther Exp Psychiatry. 1996;27(3):219-229.

53. Cusack K, Jonas DE, Forneris CA, et al. Psychological treatments for adults with posttraumatic stress disorder: a systematic review and meta-analysis. Clin Psychol Rev. 2016;43:128-141.

54. Silver SM, Brooks A, Obenchain J. Treatment of Vietnam War veterans with PTSD: a comparison of eye movement desensitization and reprocessing, biofeedback, and relaxation training. J Trauma Stress. 1995;8(2):337-342.

55. Van Eeden F. A study of dreams. PSPR. 1913;26:431-461.

56. Harb GC, Greene JL, Dent KM, Ross RJ. 1077 Lucid dreaming in veterans with PTSD: non-nightmare dreams and nightmares. Sleep. 2017;40(suppl_1):A401.

57. Dudai Y. The neurobiology of consolidations, or, how stable is the engram? Annu Rev Psychol. 2004;55(1):51-86.

58. Dębiec J, Bush DE, Ledoux JE. Noradrenergic enhancement of reconsolidation in the amygdala impairs extinction of conditioned fear in rats - a possible mechanism for the persistence of traumatic memories in PTSD. Depress Anxiety. 2011;28(3):186-193.

59. Gazarini L, Stern CA, Carobrez AP, Bertoglio LJ. Enhanced noradrenergic activity potentiates fear memory consolidation and reconsolidation by differentially recruiting $\alpha 1$ - and $\beta$-adrenergic receptors. Learn Mem. 2013;20(4):210-219.

60. Raskind MA, Dobie DJ, Kanter ED, Petrie EC, Thompson CE, Peskind ER. The alpha1-adrenergic antagonist prazosin ameliorates combat trauma nightmares in veterans with posttraumatic stress disorder: a report of 4 cases. J Clin Psychiatry. 2000;61(2):129-134. 
61. Raskind MA, Thompson C, Petrie EC, et al. Prazosin reduces nightmares in combat veterans with posttraumatic stress disorder. J Clin Psychiatry. 2002;63(7):565-568.

62. Taylor F, Raskind MA. The alpha1-adrenergic antagonist prazosin improves sleep and nightmares in civilian trauma posttraumatic stress disorder. J Clin Psychopharmacol. 2002;22(1):82-85.

63. Raskind MA, Peterson K, Williams T, et al. A trial of prazosin for combat trauma PTSD with nightmares in active-duty soldiers returned from Iraq and Afghanistan. Am J Psychiatry. 2013;170(9):1003-1010.

64. Germain A, Richardson R, Moul DE, et al. Placebo-controlled comparison of prazosin and cognitive-behavioral treatments for sleep disturbances in US Military Veterans. J Psychosom Res. 2012;72(2):89-96.

65. Khachatryan D, Groll D, Booij L, Sepehry AA, Schütz CG. Prazosin for treating sleep disturbances in adults with posttraumatic stress disorder: a systematic review and meta-analysis of randomized controlled trials. Gen Hosp Psychiatry. 2016;39:46-52.

66. Raskind MA, Peskind ER, Chow B, et al. Trial of prazosin for post-traumatic stress disorder in military veterans. $N$ Engl J Med. 2018;378(6):507-517.

67. Koola MM, Varghese SP, Fawcett JA. High-dose prazosin for the treatment of post-traumatic stress disorder. Ther Adv Psychopharmacol. 2014;4(1):43-47.

68. Morgenthaler TI, Auerbach S, Casey KR, et al. Position paper for the treatment of nightmare disorder in adults: an American Academy of Sleep Medicine position paper. J Clin Sleep Med. 2018;14(6): 1041-1055

69. Nirmalani-Gandhy A, Sanchez D, Catalano G. Terazosin for the treatment of trauma-related nightmares: a report of 4 cases. Clin Neuropharmacol. 2015;38(3):109-111.

70. Salviati M, Pallagrosi M, Valeriani G, Carlone C, Todini L, Biondi M. On the role of noradrenergic system in PTSD and related sleep disturbances. The use of terazosin in PTSD related nightmares: a case report. Clin Ter. 2013;164(2):133-137.

71. Detweiler M, Pagadala B, Candelario J, Boyle J, Detweiler J, Lutgens B. Treatment of post-traumatic stress disorder nightmares at a veterans affairs medical center. J Clin Med. 2016;5(12):117.

72. Sethi R, Vasudeva S. Doxazosin for the treatment of nightmares: does it really work? A case report. Prim Care Companion CNS Disord. 2012;14(5).

73. Roepke S, Danker-Hopfe H, Repantis D, et al. Doxazosin, an alpha1-adrenergic-receptor antagonist, for nightmares in patients with posttraumatic stress disorder and/or borderline personality disorder: a chart review. Pharmacopsychiatry. 2017;50(1):26-31.

74. Alao A, Selvarajah J, Razi S. The use of clonidine in the treatment of nightmares among patients with co-morbid PTSD and traumatic brain injury. Int J Psychiatry Med. 2012;44(2):165-169.

75. Kinzie JD, Leung P. Clonidine in Cambodian patients with posttraumatic stress disorder. J Nerv Ment Dis. 1989;177(9):546-550.

76. Boehnlein JK, Kinzie JD, Sekiya U, Riley C, Pou K, Rosborough B. A ten-year treatment outcome study of traumatized cambodian refugees. J Nerv Ment Dis. 2004;192(10):658-663.

77. Wendell K, Maxwell M. Evaluation of clonidine and prazosin for the treatment of nightime post traumatic stres disorder symptoms. Fed Pract. 2015:8-14.

78. Graham RL, Leckband S, Endow-Eyer R. PTSD nightmares: prazosin and atypical antipsychotics. Curr Psychiatr. 2012;11:59-62.

79. Schotte A, Janssen PF, Gommeren W, et al. Risperidone compared with new and reference antipsychotic drugs: in vitro and in vivo receptor binding. Psychopharmacology. 1996;124(1-2):57-73.

80. Richelson E. Receptor pharmacology of neuroleptics: relation to clinical effects. J Clin Psychiatry. 1999;60(Suppl 10):5-14.

81. David D, De Faria L, Mellman TA. Adjunctive risperidone treatment and sleep symptoms in combat veterans with chronic PTSD. Depress Anxiety. 2006;23(8):489-491.

82. Stanovic JK, James KA, Vandevere CA. The effectiveness of risperidone on acute stress symptoms in adult burn patients: a preliminary retrospective pilot study. J Burn Care Rehabil. 2001;22(3):210-213.
83. Khachiyants N, Ali R, Kovesdy CP, Detweiler JG, Kim KY, Detweiler MB. Effectiveness of risperidone for the treatment of nightmares in veterans with posttraumatic stress disorder. J Clin Psychopharmacol. 2010;30(6):735-737.

84. Jakovljević M, Sagud M, Mihaljević-Peles A. Olanzapine in the treatment-resistant, combat-related PTSD--a series of case reports. Acta Psychiatr Scand. 2003;107(5):394-396.

85. Stein MB, Kline NA, Matloff JL. Adjunctive olanzapine for SSRIresistant combat-related PTSD: a double-blind, placebo-controlled study. Am J Psychiatry. 2002;159(10):1777-1779.

86. Butterfield MI, Becker ME, Connor KM, Sutherland S, Churchill LE, Davidson JRT. Olanzapine in the treatment of post-traumatic stress disorder: a pilot study. Int Clin Psychopharmacol. 2001;16(4):197-203.

87. Deng C, Weston-Green K, Huang XF. The role of histaminergic H1 and H3 receptors in food intake: a mechanism for atypical antipsychoticinduced weight gain? Prog Neuropsychopharmacol Biol Psychiatry. 2010;34(1):1-4.

88. Weston-Green K, Huang XF, Deng C. Alterations to melanocortinergic, GABAergic and cannabinoid neurotransmission associated with olanzapine-induced weight gain. PLoS One. 2012;7(3):e33548.

89. Robert S, Hamner MB, Kose S, Ulmer HG, Deitsch SE, Lorberbaum JP. Quetiapine improves sleep disturbances in combat veterans with PTSD: sleep data from a prospective, open-label study. J Clin Psychopharmacol. 2005;25(4):387-388.

90. Britnell SR, Jackson AD, Brown JN, Capehart BP. Aripiprazole for post-traumatic stress disorder: a systematic review. Clin Neuropharmacol. 2017;40(6):273-278.

91. Lambert MT. Aripiprazole in the management of post-traumatic stress disorder symptoms in returning Global War on Terrorism veterans. Int Clin Psychopharmacol. 2006;21(3):185-187.

92. Dillard ML, Bendfeldt F, Jernigan P. Use of thioridazine in posttraumatic stress disorder. South Med J. 1993;86(11):1276-1278.

93. De Boer M, Op den Velde W, Falger PJ, Hovens JE, de Groen JH, van Duijn H. Fluvoxamine treatment for chronic PTSD: a pilot study. Psychother Psychosom. 1992;57(4):158-163.

94. Neylan TC, Metzler TJ, Schoenfeld FB, et al. Fluvoxamine and sleep disturbances in posttraumatic stress disorder. J Trauma Stress. 2001;14(3):461-467.

95. Lepkifker E, Dannon PN, Iancu I, Ziv R, Kotler M. Nightmares related to fluoxetine treatment. Clin Neuropharmacol. 1995;18(1):90-94.

96. Arora G, Sandhu G, Fleser C. Citalopram and nightmares. J Neuropsychiatry Clin Neurosci. 2012;24(2):E43.

97. Lewis JD. Mirtazapine for PTSD nightmares. Am J Psychiatry. 2002;159(11):1948-1949.

98. Mathews M, Basil B, Evcimen H, Adetunji B, Joseph S. Mirtazapineinduced nightmares. Prim Care Companion J Clin Psychiatry. 2006;8(5):311.

99. Menon V, Madhavapuri P. Low-Dose mirtazapine-induced nightmares necessitating its discontinuation in a young adult female. J Pharmacol Pharmacother. 2017;8(4):182-184.

100. Rottach KG, Schaner BM, Kirch MH, et al. Restless legs syndrome as side effect of second generation antidepressants. J Psychiatr Res. 2008;43(1):70-75.

101. Neylan TC, Lenoci M, Maglione ML, et al. The effect of nefazodone on subjective and objective sleep quality in posttraumatic stress disorder. J Clin Psychiatry. 2003;64(4):445-450.

102. Davidson JR, Weisler RH, Malik ML, Connor KM. Treatment of posttraumatic stress disorder with nefazodone. Int Clin Psychopharmacol. 1998;13(3):111-114.

103. Boehnlein JK, Kinzie JD, Ben R, Fleck J. One-year follow-up study of posttraumatic stress disorder among survivors of Cambodian concentration camps. Am J Psychiatry. 1985;142(8):956-959.

104. Warner MD, Dorn MR, Peabody CA. Survey on the usefulness of trazodone in patients with PTSD with insomnia or nightmares. Pharmacopsychiatry. 2001;34(4):128-131.

105. Harsch HH. Cyproheptadine for recurrent nightmares. Am J Psychiatry. 1986;143(11):1491-1492. 
106. Brophy MH. Cyproheptadine for combat nightmares in post-traumatic stress disorder and dream anxiety disorder. Mil Med. 1991;156(2): $100-101$.

107. Gupta S, Popli A, Bathurst E, Hennig L, Droney T, Keller P. Efficacy of cyproheptadine for nightmares associated with posttraumatic stress disorder. Compr Psychiatry. 1998;39(3):160-164.

108. Clark RD, Canive JM, Calais LA, Qualls C, Brugger RD, Vosburgh TB. Cyproheptadine treatment of nightmares associated with posttraumatic stress disorder. J Clin Psychopharmacol. 1999;19(5):486-487.

109. Abrams TE, Lund BC, Bernardy NC, Friedman MJ. Aligning clinical practice to PTSD treatment guidelines: medication prescribing by provider type. Psychiatr Serv. 2013;64(2):142-148.

110. Greenbaum MA, Neylan TC, Rosen CS. Symptom presentation and prescription of sleep medications for veterans with posttraumatic stress disorder. J Nerv Ment Dis. 2017;205(2):1-118.

111. Guina J, Rossetter SR, Derhodes BJ, Nahhas RW, Welton RS. Benzodiazepines for PTSD: a systematic review and meta-analysis. J Psychiatr Pract. 2015;21(4):281-303.

112. Braun P, Greenberg D, Dasberg H, Lerer B. Core symptoms of posttraumatic stress disorder unimproved by alprazolam treatment. J Clin Psychiatry. 1990;51(6):236-238.

113. Cates ME, Bishop MH, Davis LL, Lowe JS, Woolley TW. Clonazepam for treatment of sleep disturbances associated with combat-related posttraumatic stress disorder. Ann Pharmacother. 2004;38(9): 1395-1399.

114. Abramowitz EG, Barak Y, Ben-Avi I, Knobler HY. Hypnotherapy in the treatment of chronic combat-related PTSD patients suffering from insomnia: a randomized, zolpidem-controlled clinical trial. Int J Clin Exp Hypn. 2008;56(3):270-280.

115. Passie T, Emrich HM, Karst M, Brandt SD, Halpern JH. Mitigation of post-traumatic stress symptoms by Cannabis resin: a review of the clinical and neurobiological evidence. Drug Test Anal. 2012;4(7-8):649-659.
116. Greer GR, Grob CS, Halberstadt AL. PTSD symptom reports of patients evaluated for the New Mexico Medical Cannabis Program. $J$ Psychoactive Drugs. 2014;46(1):73-77.

117. Zuardi AW. Cannabidiol: from an inactive cannabinoid to a drug with wide spectrum of action. Rev Bras Psiquiatr. 2008;30(3):271-280.

118. Fraser GA. The use of a synthetic cannabinoid in the management of treatment-resistant nightmares in posttraumatic stress disorder (PTSD). CNS Neurosci Ther. 2009;15(1):84-88.

119. Jetly R, Heber A, Fraser G, Boisvert D. The efficacy of nabilone, a synthetic cannabinoid, in the treatment of PTSD-associated nightmares: a preliminary randomized, double-blind, placebocontrolled cross-over design study. Psychoneuroendocrinology. 2015;51:585-588

120. Jinwala FN, Gupta M. Synthetic cannabis and respiratory depression. J Child Adolesc Psychopharmacol. 2012;22(6):459-462.

121. Sweeney B, Talebi S, Toro D, et al. Hyperthermia and severe rhabdomyolysis from synthetic cannabinoids. Am J Emerg Med. 2016;34(1):121. e1-121.e2.

122. Takematsu M, Hoffman RS, Nelson LS, Schechter JM, Moran JH, Wiener SW. A case of acute cerebral ischemia following inhalation of a synthetic cannabinoid. Clin Toxicol. 2014;52(9):973-975.

123. Lapoint J, James LP, Moran CL, Nelson LS, Hoffman RS, Moran JH. Severe toxicity following synthetic cannabinoid ingestion. Clin Toxicol. 2011;49(8):760-764.

124. MacFarlane V, Christie G. Synthetic cannabinoid withdrawal: a new demand on detoxification services. Drug Alcohol Rev. 2015;34(2):147-153.

125. Rodgman CJ, Verrico CD, Worthy RB, Lewis EE. Inpatient detoxification from a synthetic cannabinoid and control of postdetoxification cravings with naltrexone. Prim Care Companion CNS Disord. 2014;16(4).

126. Degenhardt L, Hall W. Is cannabis use a contributory cause of psychosis? Can J Psychiatry. 2006;51(9):556-565.
Nature and Science of Sleep

\section{Publish your work in this journal}

Nature and Science of Sleep is an international, peer-reviewed, open access journal covering all aspects of sleep science and sleep medicine, including the neurophysiology and functions of sleep, the genetics of sleep, sleep and society, biological rhythms, dreaming, sleep disorders and therapy, and strategies to optimize healthy sleep. The manuscript

\section{Dovepress}

management system is completely online and includes a very quick and fair peer-review system, which is all easy to use. Visit http://www. dovepress.com/testimonials.php to read real quotes from published authors. 